\title{
PIECEWISE LINEARIZATION OF REAL-VALUED SUBANALYTIC FUNCTIONS
}

\author{
MASAHIRO SHIOTA
}

\begin{abstract}
We show that for a subanalytic function $f$ on a locally compact subanalytic set $X$ there exists a unique subanalytic triangulation (a simplicial complex $K$, a subanalytic homeomorphism $\pi:|K| \rightarrow X$ ) such that $\left.f \circ \pi\right|_{\sigma}$, $\sigma \in K$, are linear.
\end{abstract}

Let $X$ be a subanalytic set contained and closed in a Euclidean space. A subanalytic triangulation of $X$ is a pair $(K, \pi)$ where $K$ is a simplicial complex and $\pi$ is a subanalytic homeomorphism from the underlying polyhedron $|K|$ to $X$. Here we give $|K|$ a subanalytic structure by realizing $K$ in a Euclidean space so that $|K|$ is closed in the Euclidean space. The existence of a subanalytic triangulation of $X$ was shown by [2 and 4]. [2 and 4] use induction on the dimension of the ambient space like [5]. Moreover [10] proved the uniqueness up to PL homeomorphism, namely, that if there are two subanalytic triangulations $(K, \pi)$ and $\left(K^{\prime}, \pi^{\prime}\right)$ of $X$ then $|K|$ and $\left|K^{\prime}\right|$ are PL homeomorphic. Our purpose is the following theorem of a unique subanalytic triangulation of a subanalytic function.

Theorem 1. Let $f$ be a subanalytic function on $X$. Then there exists a unique subanalytic triangulation $(K, \pi)$ of $X$ such that for every simplex $\sigma$ in $K$, $\left.f \circ \pi\right|_{\sigma}$ is linear. Here the uniqueness means that for another subanalytic triangulation $\left(K^{\prime}, \pi^{\prime}\right)$ of $X$ with the same property as $(K, \pi)$ there exists a $P L$ homeomorphism $\tau$ from $|K|$ to $\left|K^{\prime}\right|$ such that $f \circ \pi^{\prime} \circ \tau=f \circ \pi$.

I proved this in weaker forms in [9]. I constructed $\pi$ using the integrations of vector fields. Hence triangulations of subanalytic functions in [9] were of class $C^{0}$ only, and the uniqueness did not follow because of the failure of the topological Hauptvermutung [6]. As for this paper, we apply the projection method of [5] to the local proof of Theorem 1. The uniqueness theorem [10] pastes the local subanalytic triangulations and proves globally Theorem 1 , which is similar to the proof of the theorem of $C^{\infty}$ triangulation of $C^{\infty}$ manifold (CairnsWhitehead, e.g. [7]). We use also the Alexander trick (see for its definition [8, 10] and the statement before Lemma 9). Teissier [11] also used the projection

Received by the editors September 16, 1987.

1980 Mathematics Subject Classification (1985 Revision). Primary 32B25; Secondary 32B20.

Key words and phrases. Subanalytic set, triangulation, PL function. 
method of [5] for triangulations of maps. However, for lack of uniqueness, the results are always local at the target set.

As a corollary we see that a subanalytic function is almost decided by its zero set as the germ at the zero set.

Corollary 2. Let $f_{1}, f_{2}$ be subanalytic functions on $X$ with

$$
f_{1}^{-1}(0)=f_{2}^{-1}(0), \quad\left\{f_{1}<0\right\}=\left\{f_{2}<0\right\}, \quad\left\{f_{1}>0\right\}=\left\{f_{2}>0\right\} .
$$

Then there exists a subanalytic homeomorphism $\tau$ of $X$ such that

$$
\begin{gathered}
f_{1} \circ \tau=f_{2} \quad \text { on a neighborhood of } f_{1}^{-1}(0), \\
\tau=\text { ident on } f_{1}^{-1}(0) .
\end{gathered}
$$

We can prove also some variations of Theorem 1: the nonclosed case (Remark 14), the semianalytic case (Remark 15), and the following semialgebraic case.

Theorem 3. Let $Y$ be a semialgebraic set and let $f$ be a semialgebraic bounded function on $Y$. Then there exist a finite simplicial complex $K$ and a semialgebraic homeomorphism $\pi$ from a union $Z$ of some open simplexes in $K$ to $Y$ such that $f \circ \pi$ can be extended to $|K|$ and the restriction of the extension to every simplex in $K$ is linear.

\section{COMPACT CASE}

In this section we prove Theorem 1 in the compact case in the following more general form. A simplex means always a closed simplex. For a simplex or a cell $\sigma$, Int $\sigma$ and $\partial \sigma$ mean the interior and the boundary respectively. For a simplicial complex $K$, let $K^{k}$ denote the $k$-skeleton of $K$. Let $K_{1} \subset K_{2}$ be simplicial complexes. The simplicial neighborhood of $K_{1}$ in $K_{2}$ is the smallest subcomplex of $K_{2}$ whose underlying polyhedron is a topological neighborhood of $\left|K_{1}\right|$ in $\left|K_{2}\right|$. We denote it by $N\left(K_{1}, K_{2}\right)$. We call $K_{1}$ a full subcomplex of $K_{2}$ if each simplex in $K_{2}$ with vertexes in $K_{1}$ is in $K_{1}$.

Lemma 4. For the same $f$ and $X$ as Theorem 1, assume $X$ to be compact. Let $\left\{Y_{i}\right\}$ be a finite family of subanalytic subsets of $X$. Then there exists $a$ subanalytic triangulation $(K, \pi)$ of $X$ such that for every simplex $\sigma$ in $K$, $\left.f \circ \pi\right|_{\sigma}$ is linear, and $\{\pi(\operatorname{Int} \sigma): \sigma \in K\}$ is compatible with $\left\{Y_{i}\right\}$.

Moreover, if there is another triangulation $\left(K^{\prime}, \pi^{\prime}\right)$ of $X$ with the same properties, then there exists a PL homeomorphism $\tau$ from $|K|$ to $\left|K^{\prime}\right|$ such that for every $Y_{i}$

$$
\tau\left(\pi^{-1}\left(Y_{i}\right)\right)=\pi^{\prime-1}\left(Y_{i}\right), \quad f \circ \pi^{\prime} \circ \tau=f \circ \pi .
$$

For the proof we need some concepts and some lemmas. Let $A$ be a subanalytic set in $\mathbf{R}^{n}$. A subanalytic analytic stratification $\left\{A_{i}\right\}$ of $A$ is a stratification of $A$ whose strata are subanalytic in $R^{n}$ and analytic submanifolds of $\mathbf{R}^{n}$. In this paper we assume the frontier condition of stratification, i.e. for every stratum $A_{i},\left(\overline{A_{i}} \cap A\right)-A_{i}$ is a union of strata. A family $\left\{B_{\beta}\right\}$ of subsets of $\mathbf{R}^{n}$ is compatible with another $\left\{C_{\gamma}\right\}$ if for each $\beta, \gamma, B_{\beta} \subset C_{\gamma}$ or $B_{\beta} \cap C_{\gamma}=\phi$. 
Lemma 5. Let $A, B$ be subanalytic sets contained and bounded in Euclidean space. Let $p: A \rightarrow B$ be a subanalytic map. Let $\left\{C_{k}\right\}$ be a finite family of subanalytic subsets of $A$. Then we have subanalytic analytic stratification $\left\{A_{i}\right\}$, $\left\{B_{j}\right\}$ of $A, B$, respectively, such that $\left\{A_{i}\right\}$ is compatible with $\left\{C_{k}\right\}$ and for each $A_{i}$, the restriction of $p$ to $A_{i}$ is of class analytic and a submersion to some $B_{j}$.

This follows easily from [3]. Let $A$ be a subset of $\mathbf{R}^{n}$. A point $\lambda$ of $S^{n-1}=\left\{\lambda \in \mathbf{R}^{n}:|\lambda|=1\right\}$ is singular for $A$ at a point $c$ of $\mathbf{R}^{n}$ if $A \cap(c+\mathbf{R} \lambda)$ has interior points in $c+\mathbf{R} \lambda$. We shall prove Theorem 1 by a sequence of projections $\mathbf{R}^{k} \rightarrow \mathbf{R}^{k-1}$ whose directions are not singular for some subanalytic sets. So we need to find directions which are not singular for given subanalytic sets.

Lemma 6 [5 and 11]. Let $A$ be a bounded subanalytic set in $\mathbf{R}^{n}$ of dimension $<n$. Then the set of singular points for $A$ at some points is subanalytic and of dimension $<n-1$.

Proof. Set

$$
B=A, \quad X=\left\{(a, b, \lambda) \in A \times B \times S^{n-1}: a \neq b, b-a= \pm|b-a| \lambda\right\},
$$

and let $p: X \rightarrow A \times S^{n-1}$ be the restriction to $X$ of the projection $A \times B \times$ $S^{n-1} \rightarrow A \times S^{n-1}$. Then $X$ and $p$ are subanalytic, and a point $\lambda$ of $S^{n-1}$ is singular for $A$ at a point $a$ of $A$ if and only if $\operatorname{dim} p^{-1}(a, \lambda)=1$. Let $S$ denote the set of such points $(a, \lambda)$ in $A \times S^{n-1}$.

We want to see that $S$ is subanalytic. Apply Lemma 5 to $p$. Then we have subanalytic analytic stratifications $\left\{X_{i}\right\},\left\{Y_{j}\right\}$ of $X, A \times S^{n-1}$, respectively, such that for every $X_{i}$, the restriction of $p$ to $X_{i}$ is an analytic submersion to some $Y_{j}$. Here we can assume that all strata are connected. Then for each $i$

$$
\operatorname{rank} d\left(\left.p\right|_{X_{i}}\right)=\text { const . }
$$

Hence $S$ is the image under $p$ of the union of $X_{i}$ 's such that

$$
\operatorname{rank} d\left(\left.p\right|_{X_{i}}\right) \neq \operatorname{dim} X_{i} \text {. }
$$

Now recall the fact [3] that the image of a bounded subanalytic set under a subanalytic map is subanalytic. It follows from this fact that $S$ is subanalytic.

Apply the above fact to $S$ and the projection $p^{\prime}: A \times S^{n-1} \rightarrow S^{n-1}$ again. Then the image $p^{\prime}(S)$ is subanalytic. Clearly $p^{\prime}(S)$ coincides with the set of singular points for $A$ at some points. For the proof of $\operatorname{dim} p^{\prime}(S)<n-1$, we need Lemma 3 in [5], which states that $p^{\prime}(S)$ is meager in $S^{n-1}$ if $A$ is the zero set of an analytic function defined on an open set in $\mathbf{R}^{n}$. This statement holds true for general $A$ because by a subanalytic analytic stratification we can describe $A$ as a finite union of analytic submanifolds of $\mathbf{R}^{n}$. If $\operatorname{dim} p^{\prime}(S)=$ $n-1$ then $p^{\prime}(S)$ would have inner points in $S^{n-1}$, which is a contradiction. Hence the above inequality holds, which completes the proof. 
Let us consider the parameterized case of Lemma 6 . Let $A_{t}, t \in \mathbf{R}$, be a family of subanalytic sets in $\mathbf{R}^{n}$ of dimension $<n$. Assume that the set

$$
A=\left\{(a, t) \in \mathbf{R}^{n} \times \mathbf{R}: a \in A_{t}\right\}
$$

is a bounded subanalytic set in $\mathbf{R}^{n+1}$. For every $t \in R$, let $T_{t} \subset S^{n-1}$ denote the set of singular points for $A_{t}$ at some points in $\mathbf{R}^{n}$. Set

$$
T=\left\{(\lambda, t) \in S^{n-1} \times \mathbf{R}: \lambda \in T_{t}\right\} .
$$

Lemma 7 [5 and 11]. $T$ is subanalytic, and for every $t \in \mathbf{R}$

$$
\operatorname{dim} \bar{T} \cap S^{n-1} \times t<n-1,
$$

where $\bar{T}$ means the closure of $T$ in $S^{n-1} \times \mathbf{R}$.

Proof. Replace $X$ in the proof of Lemma 6 by

$$
\left\{\left(a, t, b, t^{\prime}, \lambda\right) \in A \times B \times S^{n-1}: a \neq b, t=t^{\prime}, b-a= \pm|b-a| \lambda\right\}
$$

and replace $p^{\prime}$ by the restriction $q$ to $A \times S^{n-1}$ of the composition of the projection

$$
\mathbf{R}^{n} \times \mathbf{R} \times S^{n-1} \rightarrow \mathbf{R} \times S^{n-1}
$$

with the replacement $\mathbf{R} \times S^{n-1} \rightarrow S^{n-1} \times \mathbf{R}$. Then the proof of Lemma 6 proceeds, and we see that $T=q(S)$ and $T$ is subanalytic and of dimension $<n$. Recall the following fact [3]. Let $C$ be a subanalytic set in $\mathbf{R}^{n}$. Then

$$
\operatorname{dim} \bar{C}=\operatorname{dim} C, \quad \operatorname{dim}(\bar{C}-C)<\operatorname{dim} C .
$$

Hence for every $t \in \mathbf{R}$,

$$
\bar{T} \cap S^{n-1} \times t=\bar{T} \times t \cup\left(\left(\overline{T-S^{n-1} \times t}\right)-\left(T-S^{n-1} \times t\right)\right)
$$

is of dimension $<n-1$.

Lemma 8. Let $V$ be a compact subanalytic set in $\mathbf{R}^{n} \times \mathbf{R}$, and let $\left\{W_{i}\right\}$ be a finite family of subanalytic sets in $\mathbf{R}^{n} \times \mathbf{R}$. Then there exist $\varepsilon>0$, a finite simplicial complex $K$ in $\mathbf{R}^{n} \times \mathbf{R}$ and a subanalytic homeomorphism $\tau$ from $|K|$ to

$$
V(\varepsilon)=\left\{(x, t) \in V \subset \mathbf{R}^{n} \times \mathbf{R}:|t| \leq \varepsilon\right\}
$$

of the form

$$
\tau(x, t)=\left(\tau^{\prime}(x, t), t\right) \text { for }(x, t) \in|K| \subset \mathbf{R}^{n} \times \mathbf{R}
$$

such that $\{\tau(\operatorname{Int} \sigma): \sigma \in K\}$ is compatible with $\left\{W_{i}\right\}$.

Proof. We prove the lemma by induction on $n$. If $n=0$ this is trivial. So assume the lemma for $n-1$. Let $\left\{V_{j}\right\}$ be a subanalytic analytic stratification of $V$ compatible with $\left\{W_{i}\right\}$. Let $A$ denote the union of all $V_{j}$ of dimension $\leq n$ with

$$
\operatorname{dim}\left(V_{j} \cap \mathbf{R}^{n} \times 0\right)<n .
$$

For every $t \in \mathbf{R}$, set

$$
A_{t}=\left\{x \in \mathbf{R}^{n}:(x, t) \in A\right\},
$$


and define $T_{t}$ and $T$ for these $A_{t}, t \in \mathbf{R}$, as we did just before Lemma 7 . Then after changing the coordinate system of $\mathbf{R}^{n}$ we can assume by Lemma 7 $(0, \ldots, 0,1,0) \notin \bar{T}$, which implies $(0, \ldots, 1) \notin T_{t}$ for every $t \in \mathbf{R}$ with $|t| \leq \varepsilon_{0}$ for some $\varepsilon_{0}>0$. For simplicity of notations we assume this condition for all $t$. Let $p$ denote the restriction to $A$ of the projection

$$
\mathbf{R}^{n} \times \mathbf{R} \ni\left(x_{1}, \ldots, x_{n}, t\right) \rightarrow\left(x_{1}, \ldots, x_{n-1}, t\right) \in \mathbf{R}^{n-1} \times \mathbf{R},
$$

and apply Lemma 5 to $p$ and $\left\{V_{j}\right\}$. Then we have subanalytic analytic stratifications $\left\{A_{k}\right\},\left\{B_{l}\right\}$ of $A, p(A)$, respectively, such that for each $A_{k},\left.p\right|_{A_{k}}$ is an analytic submersion to some $B_{l}$ and that $\left\{A_{k}\right\}$ is compatible with $\left\{V_{j}\right\}$. Here we note that $\left.p\right|_{A_{k}}$ is surjective to $B_{l}$ since $A$ is compact and that $\left\{B_{l}\right\}$ is compatible with $\left\{p\left(V_{j}\right)\right\}$ by the frontier condition of stratification. Hence it follows from the nonsingularity of $(0, \ldots, 0,1,0)$ for $A$ that $\left.p\right|_{A_{k}}$ is a homeomorphism onto $B_{l}$.

By the induction hypothesis we can assume that $p(A)$ is a polyhedron in $\mathbf{R}^{n-1} \times \mathbf{R}$ and each $\bar{B}_{l}$ is a subpolyhedron. Let $L$ be a simplicial complex in $\mathbf{R}^{n-1} \times \mathbf{R}$ with underlying polyhedron $=p(A)$ such that each $B_{l}$ is a union of Int $\sigma$ for some simplexes $\sigma$ in $L$. Let $\widetilde{L}$ denote the family of sets of the form $\overline{A_{k}} \cap p^{-1}(\sigma)$ for $A_{k}$ and $\sigma \in L$ with $p\left(A_{k}\right) \supset \operatorname{Int} \sigma$.

We want to see the following. For every

$$
\tilde{\sigma}=\overline{A_{k}} \cap p^{-1}(\sigma) \in \widetilde{L},
$$

the restriction of $p$ to $\tilde{\sigma}$ is a subanalytic homeomorphism onto $\sigma$. It is clearly surjective. Moreover we can prove its injectivity in the same way as Hardt, Trans. Amer. Math. Soc. 211 (1975), Theorem 3.1(2), as follows. Assume it to be not injective, i.e. there exist $x \neq y \in \tilde{\sigma}$ with $p(x)=p(y)$. Set

$$
\tilde{\sigma}_{0}=p^{-1}(\operatorname{Int} \sigma) \cap \tilde{\sigma} .
$$

Then $\left.p\right|_{\tilde{\sigma}_{0}}$ is a homeomorphism onto Int $\sigma$, and $p\left(\tilde{\sigma}-\tilde{\sigma}_{0}\right)=\partial \sigma$. Hence $x, y \in \tilde{\sigma}-\tilde{\sigma}_{0}, p(x) \in \partial \sigma$. As $\overline{\tilde{\sigma}}_{0}=\tilde{\sigma}$, there exists a subanalytic curve $\varphi:[0,1] \rightarrow \tilde{\sigma}$ such that $\varphi(0)=x, \varphi(1)=y, \varphi((0,1)) \subset \tilde{\sigma}_{0}$. We can contract $p \circ \varphi$ to the point $p(x)$ in $\sigma$ since $\sigma$ is a simplex. Hence we have subanalytic curves

$$
\varphi_{s}:[0,1] \rightarrow \tilde{\sigma}, \quad s \in[0,1),
$$

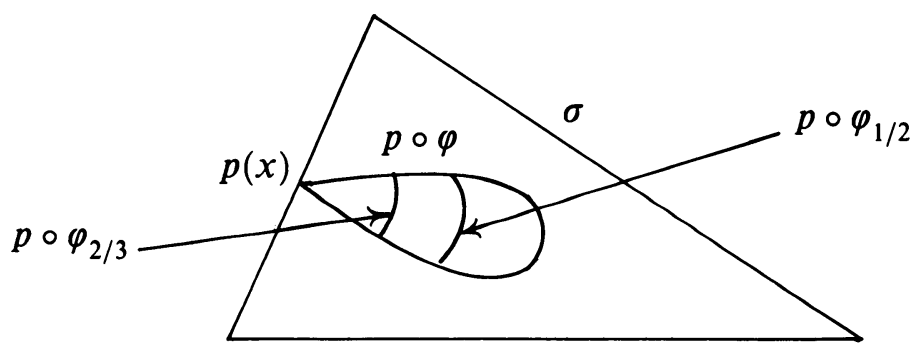


such that $\varphi_{0}=\varphi$, for each $s \in[0,1)$

$$
\varphi_{s}=\varphi \text { on a neighborhood of }\{0,1\} \text {, }
$$

and $p \circ \varphi_{s}([0,1])$ contracts to $p(x)$ as $s \rightarrow 1$. Let $P$ be a hypersurface in $\mathbf{R}^{n} \times \mathbf{R}$ which separates $x$ and $y$, and set

$$
Q=\bigcap_{s \in[0,1)} \overline{P \cap \bigcup_{s \leq s^{\prime}} \varphi_{s^{\prime}}([0,1])}
$$

Then $Q$ is not empty because $\tilde{\sigma}$ is compact, and $Q \subset P, p(Q)=p(x)$. However, $p^{-1}(p(x))$ is a finite set, and then we can choose $P$ so that it does not contain any point of $p^{-1}(p(x))$, which is a contradiction. Thus $\left.p\right|_{\tilde{\sigma}}: \tilde{\sigma} \rightarrow \sigma$ is a homeomorphism.

Assume $V=A$. For every $\tilde{\sigma} \in \widetilde{L}$ we will slide $\tilde{\sigma}$ to a simplex parallel to the $x_{n}$-axis, and then we will define $K$ as a family of such simplexes. We call a point of $\tilde{\sigma} \in \tilde{L}$ a vertex if its image under $p$ is a vertex in $L$. For every $\tilde{\sigma} \neq \tilde{\sigma}^{\prime} \in \widetilde{L}$ with the same image $\sigma$ under $p$, their interiors

$$
\tilde{\sigma} \cap p^{-1}(\operatorname{Int} \sigma), \quad \tilde{\sigma}^{\prime} \cap p^{-1}(\operatorname{Int} \sigma)
$$

do not intersect, however their sets of vertexes may coincide. Hence in general we can not distinguish $\tilde{\sigma}$ and $\tilde{\sigma}^{\prime}$ by their vertexes. So replace $L$ by its barycentric subdivision. Then we can distinguish them. Moreover, for the vertexes

$$
\left.\begin{array}{l}
a_{i}=\left(x_{1 i}, \ldots, x_{n i}, t_{i}\right) \\
a_{i}^{\prime}=\left(x_{1 i}^{\prime}, \ldots, x_{n i}^{\prime}, t_{i}^{\prime}\right)
\end{array}\right\} \in \mathbf{R}^{n} \times \mathbf{R}, \quad i=0, \ldots, m,
$$

of $\tilde{\sigma}, \tilde{\sigma}^{\prime}$, respectively, with $p\left(a_{i}\right)=p\left(a_{i}^{\prime}\right)$, i.e. $x_{j i}=x_{j i}^{\prime}, j \neq n, t_{i}=t_{i}^{\prime}$, we have

$$
x_{n i} \leq x_{n i}^{\prime}, \quad i=0, \ldots, m
$$

or

$$
x_{n i} \geq x_{n i}^{\prime}, \quad i=0, \ldots, m,
$$

and in each case at least one strict inequality holds.

Let $K$ denote the simplicial complex consisting of simplexes spanned by the vertexes of $\tilde{\sigma} \in \widetilde{L}$, and for every $\delta \in K$ with vertexes $=$ the vertexes of $\tilde{\sigma} \in \tilde{L}$, define a subanalytic homeomorphism $\tau_{\delta}: \delta \rightarrow \tilde{\delta}$ so that $p \circ \tau_{\delta}(x)=$ $p(x)$, namely, $\tau$ is a slide parallel to the $x_{n}$-axis. Then the correspondence $K \ni \delta \rightarrow \tilde{\sigma} \in \widetilde{L}$ is bijective, and $\tau_{\delta}$ are compatible each other, to be precise,

$$
\left.\tau_{\delta}\right|_{\delta \cap \delta^{\prime}}=\left.\tau_{\delta^{\prime}}\right|_{\delta \cap \delta^{\prime}}=\tau_{\delta \cap \delta^{\prime}} \text { for } \delta, \delta^{\prime} \in K .
$$

Therefore we can define a subanalytic homeomorphism $\tau$ from $|K|$ to $V$ as $\tau_{\delta}$ on each $\delta \in K$, which proves Lemma 8 in the case of $V=A$.

Assume $V \neq A$. Define $K_{1}$ and $\tau_{1}:\left|K_{1}\right| \rightarrow A$ for $A$ as we did $K$ and $\tau$ in the case of $V=A$. Extend $\tau_{1}^{-1}$ to $V \rightarrow \mathbf{R}^{n} \times \mathbf{R}$ as follows. For each

$$
x=\left(x_{1}, \ldots, x_{n}, t\right) \in V-A \text {. }
$$


There exist unique $x_{n}^{\prime}<x_{n}<x_{n}^{\prime \prime}$ such that

$$
\begin{aligned}
& x^{\prime}=\left(x_{1}, \ldots, x_{n-1}, x_{n}^{\prime}, t\right), \quad x^{\prime \prime}=\left(x_{1}, \ldots, x_{n-1}, x_{n}^{\prime \prime}, t\right) \in A, \\
& \left(x_{1}, \ldots, x_{n-1}, y, t\right) \in V-A \text { for any } x_{n}^{\prime}<y<x_{n}^{\prime \prime} .
\end{aligned}
$$

Set

$$
\tau^{-1}(x)=\tau_{1}^{-1}\left(x^{\prime}\right)\left(x_{n}^{\prime \prime}-x_{n}\right) /\left(x_{n}^{\prime \prime}-x_{n}^{\prime}\right)+\tau_{1}^{-1}\left(x^{\prime \prime}\right)\left(x_{n}-x_{n}^{\prime}\right) /\left(x_{n}^{\prime \prime}-x_{n}^{\prime}\right) .
$$

Then $\tau^{-1}$ is a well-defined subanalytic injection from $V$ to $\mathbf{R}^{n} \times \mathbf{R}$. The image is a union of $\left|K_{1}\right|$ and some connected components of $\mathbf{R}^{n} \times \mathbf{R}-\left|K_{1}\right|$ or $\mathbf{R}^{n} \times 0-\left|K_{1}\right|$, consequently, the image is a polyhedron. Let $K$ denote rectilinear triangulation of $\tau^{-1}(V)$ which is a subdivision of $K_{1}$ on $\left|K_{1}\right|$ and triangulates $\tau^{-1}(V) \cap \mathbf{R}^{n} \times 0$. Then $K$ and $\tau:|K| \rightarrow V$ are what we wanted, which proves the lemma.

We shall need generalizations (Lemmas 9, 10) of the uniqueness theorem of subanalytic triangulation [10]. First we explain a concept and the Alexander trick. Let $\varphi$ be a subanalytic homeomorphism from a subanalytic set $X$ to another $Y$. A subanalytic isotopy $\varphi_{t}, t \in[0,1]$, of $\varphi$ is a family of homeomorphisms from $X$ to $Y$ such that $\varphi_{0}=\varphi$ and $X \times[0,1] \ni(x, t) \rightarrow \varphi_{t}(x) \in Y$ is subanalytic.

Let $X, Y$ be compact polyhedrons, $p, q$ be points, and $p * X, q * Y$ be the cones. Let $h: p * X \rightarrow q * Y$ be a subanalytic homeomorphism whose restriction to the base $X$ is PL onto the base $Y$. Then there exists a subanalytic isotopy $h_{t}, t \in[0,1]$, of $h$ such that $h_{t}=h$ on $X, t \in[0,1]$, and $h_{1}$ is PL.

For the same $X, Y, p$ and $q$, let $G: p * X \rightarrow q * Y$ be a subanalytic homeomorphism with $G(X)=Y$. Let $g_{t}, t \in[0,1]$, be a subanalytic isotopy of $\left.G\right|_{X}$. Then there exists a subanalytic isotopy $G_{t}, t \in[0,1]$, of $G$ such that $\left.G_{t}\right|_{X}=g_{t}, t \in[0,1]$.

We can construct these $h_{t}$ and $G_{t}$ by the method known as the Alexander trick (see [8 and 10]). However we call the results themselves the Alexander trick in this paper.

Lemma 9. Let $K$ be a finite simplicial complex, and let $X$ be a polyhedron. Let $\varphi$ be a subanalytic homeomorphism from $|K|$ to $X$ such that for every $\sigma \in K, \varphi(\sigma)$ is a subpolyhedron of $X$. Then there exists a subanalytic isotopy $\varphi_{t}, t \in[0,1]$, of $\varphi$ such that $\varphi_{1}$ is PL and for every $\sigma \in K$ and $t \in[0,1]$, $\varphi_{t}(\sigma)=\varphi(\sigma)$.

Proof. Let $n=\operatorname{dim} K$. We prove the lemma by induction on $n$. If $n=0$ it is trivial. Hence assume it for $K^{n-1}$, i.e. a subanalytic isotopy $\varphi_{t}^{n-1}, t \in[0,1]$, of $\left.\varphi\right|_{\left|K^{n-1}\right|}$ with the required properties. Let $\sigma$ be a $n$-simplex in $K$. It suffices to extend $\varphi_{t}^{n-1}$ to $\sigma$, moreover, to consider the case where $K$ consists of $\sigma$ and its faces.

Now the uniqueness theorem of subanalytic triangulation implies that $\varphi(\sigma)$ is a PL disk. Hence by the latter half of the Alexander trick there exists a 
subanalytic isotopy $\psi_{t}, t \in[0,1]$, of $\varphi$ such that $\left.\psi_{t}\right|_{\partial \sigma}=\varphi_{t}^{n-1}, t \in[0,1]$. Next, apply the first half to $\psi_{1}$. Then there exists a subanalytic isotopy $\psi_{t}^{\prime}$, $t \in[0,1]$, of $\psi_{1}$ such that

$$
\psi_{t}^{\prime}=\psi_{1} \quad \text { on } \partial \sigma, \quad t \in[0,1],
$$

and $\psi_{1}^{\prime}$ is PL. Hence the isotopy $\varphi_{t}, t \in[0,1]$, of $\varphi$ defined by

$$
\varphi_{t}= \begin{cases}\psi_{2 t} & \text { for } t \in\left[0, \frac{1}{2}\right], \\ \psi_{2 t-1}^{\prime} & \text { for } t \in\left[\frac{1}{2}, 1\right],\end{cases}
$$

is what we wanted.

Lemma 10. Let $X, Y$ be compact polyhedrons, and let $\left\{X_{i}\right\},\left\{Y_{i}\right\}$ be finite families of subpolyhedrons of $X, Y$ respectively. Let $\varphi$ be a subanalytic homeomorphism from $X$ to $Y$ whose image of every $X_{i}$ is $Y_{i}$. Then there exists a subanalytic isotopy $\varphi_{t}, t \in[0,1]$, of $\varphi$ such that $\varphi_{1}$ is $P L$ and for every $X_{i}$ and $t \in[0,1], \varphi_{t}\left(X_{i}\right)=Y_{i}$.

Proof. Let $K_{1}, K_{2}$ be rectilinear triangulations of $X, Y$ whose open simplex sets are compatible with $\left\{X_{i}\right\},\left\{Y_{i}\right\}$ respectively. By the triangulation theorem of subanalytic set $[2,4]$ or Lemma 8 we have a simplicial complex $L$ and a subanalytic homeomorphism $\psi$ from $|L|$ to $X$ such that $\{\psi(\operatorname{Int} \sigma): \sigma \in L\}$ is compatible with

$$
K_{1} \cup\left\{\varphi^{-1}(\sigma): \sigma \in K_{2}\right\} .
$$

Apply Lemma 9 to $K_{1}, \psi^{-1}: X \rightarrow|L|$ and $K_{2},(\varphi \circ \psi)^{-1}: Y \rightarrow|L|$. Then there exist subanalytic isotopies $\alpha_{t}, \beta_{t}, t \in[0,1]$, of $\psi^{-1},(\varphi \circ \psi)^{-1}$, respectively, such that $\alpha_{1}, \beta_{1}$ are PL and

$$
\begin{aligned}
& \alpha_{t}(\sigma)=\psi^{-1}(\sigma) \text { for } \sigma \in K_{1}, t \in[0,1], \\
& \beta_{t}(\delta)=(\varphi \circ \psi)^{-1}(\delta) \text { for } \delta \in K_{2}, t \in[0,1] .
\end{aligned}
$$

In particular, for all $i$ and $t \in[0,1]$

$$
\alpha_{t}\left(X_{i}\right)=\psi^{-1}\left(X_{i}\right)=(\varphi \circ \psi)^{-1}\left(Y_{i}\right)=\beta_{t}\left(Y_{t}\right) .
$$

Hence the compositions $\beta_{t}^{-1} \circ \alpha_{t}, t \in[0,1]$, satisfy the requirements.

Recall a fact of PL topology which will be used in the following proof (e.g., see the proof of 3.11 in [8]). Let $f$ be a function on $|K|$ for a simplicial complex $K$ whose restriction to every simplex is linear. Assume that $[0,1]$ does not contain the values of $f$ at vertexes of $K$. Then there exist a simplicial complex $L$ and a PL homeomorphism $\psi$ from $|L| \times[0,1]$ to $f^{-1}[0,1]$ such that

$$
f \circ \psi=\text { the projection on }[0,1] \text {, }
$$

and for every $\sigma \in K, \psi^{-1}(\sigma)$ is the product of a union of some simplexes in $L$ and $[0,1]$. 
Proof of the first half of Lemma 4. We can assume that $Y_{i}$ are closed in $X$. For $a<b \in \mathbf{R}$, set

$$
X(a, b)=f^{-1}((a, b)), \quad X[a, b]=f^{-1}([a, b]), \quad X[a]=f^{-1}(a) .
$$

Apply Lemma 8 to the graph of $f$. Then there exist closed intervals $\left[a_{j}, b_{j}\right]$, $j=1, \ldots, m$, in $\mathbf{R}$, simplicial complexes $K_{j}, j=1, \ldots, m$, and subanalytic homeomorphisms

$$
\pi_{j}:\left|K_{j}\right| \rightarrow X\left[a_{j}, b_{j}\right], \quad j=1, \ldots, m,
$$

such that $\left.f \circ \pi_{j}\right|_{\sigma}, \sigma \in K_{j}$, are all linear, $\left\{\pi_{j}(\right.$ Int $\left.\sigma): \sigma \in K_{j}\right\}$ are all compatible with $\left\{Y_{i}\right\}$, and $\left\{\left(a_{j}, b_{j}\right)\right\}_{j=1, \ldots, m}$ is a covering of the value set of $f$. We want to paste these subanalytic triangulations together. We can assume

$$
m=2, \quad\left[a_{1}, b_{1}\right]=[0,4], \quad\left[a_{2}, b_{2}\right]=[1,5] \quad(\text { i.e. } X=X[0,4] \cup X[1,5])
$$

and that $X[2,3]$ does not contain the images of vertexes of $K_{1}, K_{2}$ under $\pi_{1}$, $\pi_{2}$ respectively.

By the fact stated before this proof, there exist simplicial complexes $L_{1}, L_{2}$ and PL homeomorphisms

$$
\psi_{j}:\left|L_{j}\right| \times[2,3] \rightarrow \pi_{j}^{-1}(X[2,3]), \quad j=1,2,
$$

such that

$$
f \circ \pi_{j} \circ \psi_{j}=\text { the projection on }[2,3]
$$

and for every $\sigma \in K_{j}, j=1,2, \psi_{j}^{-1}(\sigma)$ is the product of a union of some simplexes in $L_{j}$ and [2,3]. Define subpolyhedrons $A_{i j}$ of $\left|L_{j}\right|$ by

$$
A_{i j} \times[2,3]=\left(\pi_{j} \circ \psi_{j}\right)^{-1}\left(Y_{i}\right) \text { for all } i, j .
$$

Then

$$
\pi_{1} \circ \psi_{1}\left(A_{i 1} \times t\right)=\pi_{2} \circ \psi_{2}\left(A_{i 2} \times t\right), \quad t \in[2,3] .
$$

Consider the subanalytic homeomorphism $\varphi$ from $\left|L_{1}\right|$ to $\left|L_{2}\right|$ defined by

$$
\pi_{2} \circ \psi_{2}(\varphi(x), 3)=\pi_{1} \circ \psi_{1}(x, 3),
$$

note that $\varphi$ maps each $A_{i 1}$ to $A_{i 2}$, and apply Lemma 10 to $\varphi,\left\{A_{i 1}\right\},\left\{A_{12}\right\}$. Then there exists a subanalytic isotopy $\varphi_{t}, t \in[0,1]$, of $\varphi$ such that $\varphi_{1}$ is PL and for every $A_{i 1}$ and $t \in[0,1], \varphi_{t}\left(A_{i 1}\right)=A_{i 2}$.

Set

$$
\begin{aligned}
& \Phi_{2+t}=\varphi^{-1} \circ \varphi_{t}, \quad t \in[0,1], \\
& \psi_{1}^{\prime}(x, t)=\psi_{1}\left(\Phi_{t}(x), t\right) \text { for }(x, t) \in\left|L_{1}\right| \times[2,3] .
\end{aligned}
$$

Then $\psi_{1}^{\prime}$ is a subanalytic homeomorphism from $\left|L_{1}\right| \times[2,3]$ to $\pi_{1}^{-1}(X[2,3])$ such that

$$
\begin{aligned}
& f \circ \pi_{1} \circ \psi_{1}^{\prime}=\text { the projection on }[2,3], \\
& A_{i 1} \times[2,3]=\left(\pi_{1} \circ \psi_{1}^{\prime}\right)^{-1}\left(Y_{i}\right) \text { for every } i, \\
& \psi_{1}^{\prime}=\psi_{1} \text { on }\left|L_{1}\right| \times 2 .
\end{aligned}
$$


We have

$$
\begin{aligned}
& \left(\pi_{2} \circ \psi_{2}\right)^{-1} \circ \pi_{1} \circ \psi_{1}^{\prime}(x, 3)=\left(\pi_{2} \circ \psi_{2}\right)^{-1} \circ \pi_{1} \circ \psi_{1} \circ \psi_{1}^{-1} \circ \psi_{1}^{\prime}(x, 3) \\
& \quad=\left(\pi_{2} \circ \psi_{2}\right)^{-1} \circ \pi_{1} \circ \psi_{1}\left(\varphi^{-1} \circ \varphi_{1}(x), 3\right)=\left(\varphi \circ \varphi^{-1} \circ \varphi_{1}(x), 3\right) \\
& \quad=\left(\varphi_{1}(x), 3\right) .
\end{aligned}
$$

Hence

$$
\left.\left(\pi_{2} \circ \psi_{2}\right)^{-1} \circ \pi_{1} \circ \psi_{1}^{\prime}\right|_{\left|L_{1}\right| \times 3} \text { and }\left.\pi_{2}^{-1} \circ \pi_{1} \circ \psi_{1}^{\prime}\right|_{\left|L_{1}\right| \times 3}
$$

are PL.

Let $Z$ denote the quotient polyhedron of the disjoint union of $\pi_{1}^{-1}(X[0,2])$, $\left|L_{1}\right| \times[2,3]$ and $\pi_{2}^{-1}(X[3,5])$ by the equivalence relation of the PL homeomorphisms

$$
\psi_{1}:\left|L_{1}\right| \times 2 \rightarrow \pi_{1}^{-1}(X[2]), \quad \pi_{2}^{-1} \circ \pi_{1} \circ \psi_{1}^{\prime}:\left|L_{1}\right| \times 3 \rightarrow \pi_{2}^{-1}(X[3]) .
$$

Define a subanalytic homeomorphism $\pi$ from $Z$ to $X$ by

$$
\pi= \begin{cases}\pi_{1} & \text { on } \pi_{1}^{-1}(X[0,2]), \\ \pi_{1} \circ \psi_{1}^{\prime} & \text { on }\left|L_{1}\right| \times[2,3], \\ \pi_{2} & \text { on } \pi_{2}^{-1}(X[3,5]) .\end{cases}
$$

Then each $\pi^{-1}\left(Y_{i}\right)$ and $f \circ \pi$ are polyhedrons and PL, respectively, in each of $\pi_{1}^{-1}(X[0,2]),\left|L_{1}\right| \times[2,3], \pi_{2}^{-1}(X[3,5])$. Hence there exists a rectilinear triangulation of $Z$ whose open simplex set is compatible with $\left\{\pi^{-1}\left(Y_{i}\right)\right\}$ such that the restriction of $f \circ \pi$ to every simplex is linear. This completes the proof.

Lemma 11. Let $K$ be a simplicial complex with underlying polyhedron $X$. Let $f_{1}, f_{2}$ be functions on $X$ such that for every $\sigma \in K,\left.f_{1}\right|_{\sigma}$ and $\left.f_{2}\right|_{\sigma}$ are linear,

$$
f_{1}^{-1}(0)=f_{2}^{-1}(0)=Y, \quad\left\{f_{1}>0\right\}=\left\{f_{2}>0\right\}, \quad\left\{f_{1}<0\right\}=\left\{f_{2}<0\right\} .
$$

Then for any neighborhood $U$ of $Y$ in $X$ there exists a PL homeomorphism $\pi$ of $X$ such that

$$
\begin{aligned}
& f_{1} \circ \pi=f_{2} \quad \text { on a neighborhood of } Y \text { in } X, \\
& \pi=\text { ident } \quad \text { on } Y \cup U^{c}, \\
& \pi(\sigma)=\sigma, \quad \sigma \in K .
\end{aligned}
$$

Proof. Let $K_{1}$ denote the subcomplex of $K$ such that $\left|K_{1}\right|=Y$. Choosing a subdivision of $K$ if necessary, we assume $U=\left|N\left(K_{1}, K\right)\right|$. We construct $\pi$ on $\left|K^{k}\right|$ by induction on $k$ in the same way as the proof of Lemma 9 . Hence we can reduce the problem to the following.

Let $\sigma$ be a simplex with vertexes $a_{0}, \ldots, a_{n}$. Let $f_{1}, f_{2}$ be linear functions on $\sigma$ such that for $i=1,2$

$$
f_{i}\left(a_{j}\right) \begin{cases}=0, & j=0, \ldots, m, \\ >0, & j=m+1, \ldots, n .\end{cases}
$$


Let $\delta_{1}$ and $\delta_{2}$ denote the faces of $\sigma$ spanned by $a_{0}, \ldots, a_{m}$ and $a_{m+1}, \ldots, a_{n}$ respectively. Let $\pi^{\prime}$ be a PL homeomorphism of $\partial \sigma$ such that

$$
\begin{aligned}
& \pi^{\prime}=\text { ident } \text { on } \delta_{1} \cup \delta_{2}, \\
& f_{1} \circ \pi^{\prime}=f_{2} \text { on a neighborhood of } \delta_{1} \text { in } \partial \sigma .
\end{aligned}
$$

Then there exist a PL homeomorphism extension $\pi$ of $\pi^{\prime}$ to $\sigma$ such that

$$
f_{1} \circ \pi=f_{2} \text { on a neighborhood of } \delta_{1} \text { in } \sigma .
$$

Proof of this statement. Let $b_{1}, b_{2}$ be points in Int $\sigma$ such that $f_{1}\left(b_{1}\right)=f_{2}\left(b_{2}\right)$. Then the PL map $\pi: \sigma \rightarrow \sigma$, defined by

$$
\pi\left(\lambda x+(1-\lambda) b_{2}\right)=\lambda \pi^{\prime}(x)+(1-\lambda) b_{1}, \quad x \in \partial \sigma, 0 \leq \lambda \leq 1,
$$

satisfies the requirements.

Proof of the latter half of Lemma 4 . We shall use a reduction to an easy case by stages. Apply the first half to $f, X$ and

$$
A=\{\pi(\sigma): \sigma \in K\} \cup\left\{\pi^{\prime}\left(\sigma^{\prime}\right): \sigma^{\prime} \in K^{\prime}\right\} .
$$

Then there exists a third subanalytic triangulation $\left(K^{\prime \prime}, \pi^{\prime \prime}\right)$ of $X$ such that for every simplex $\sigma^{\prime \prime}$ of $K^{\prime \prime},\left.f \circ \pi^{\prime \prime}\right|_{\sigma^{\prime \prime}}$ is linear, and $\left\{\pi^{\prime \prime}\left(\operatorname{Int} \sigma^{\prime \prime}\right): \sigma^{\prime \prime} \in K^{\prime \prime}\right\}$ is compatible with $A$. For the proof it suffices to find PL homeomorphisms $\tau:|K| \rightarrow\left|K^{\prime \prime}\right|, \tau^{\prime}:\left|K^{\prime}\right| \rightarrow\left|K^{\prime \prime}\right|$ such that

$$
\begin{gathered}
\tau(\sigma)=\pi^{\prime \prime-1} \circ \pi(\sigma), \quad \sigma \in K, \\
\tau^{\prime}\left(\sigma^{\prime}\right)=\pi^{\prime \prime-1} \circ \pi^{\prime}\left(\sigma^{\prime}\right), \quad \sigma^{\prime} \in K^{\prime}, \\
f \circ \pi^{\prime \prime} \circ \tau=f \circ \pi \quad \text { on }|K|, \\
f \circ \pi^{\prime \prime} \circ \tau^{\prime}=f \circ \pi^{\prime} \quad \text { on }\left|K^{\prime}\right| .
\end{gathered}
$$

Hence we reduce the problem to the case where $X=\left|K^{\prime}\right|, \pi^{\prime}=$ ident, and the open simplex set of $K^{\prime}$ is compatible with $\{\pi(\sigma): \sigma \in K\}$, and we need only to choose a PL homeomorphisms $\tau$ from $|K|$ to $\left|K^{\prime}\right|$ so that

$$
\tau(\sigma)=\pi(\sigma), \quad \sigma \in K, \quad f \circ \tau=f \circ \pi \quad \text { on }|K| .
$$

Let $a_{1}<a_{2}<\cdots$ be the value set of $K^{\prime 0}$ under $f$, and set for all $i$

$$
\begin{aligned}
& B_{i}^{\prime}=f^{-1}\left(\left[a_{i}, a_{i+1}\right]\right), \quad C_{i}^{\prime}=f^{-1}\left(a_{i}\right), \\
& B_{i}=\pi^{-1}\left(B_{i}^{\prime}\right), \quad C_{i}=\pi^{-1}\left(C_{i}^{\prime}\right) .
\end{aligned}
$$

Then $B_{i}, B_{i}^{\prime}$ are subpolyhedrons. Apply Lemma 10 to

$$
\pi:|K| \rightarrow\left|K^{\prime}\right|, \quad\left\{B_{i}\right\} \cup K, \quad\left\{B_{i}^{\prime}\right\} \cup\{\pi(\sigma): \sigma \in K\} .
$$

Then we have a PL homeomorphism $\mu$ from $|K|$ to $\left|K^{\prime}\right|$ such that

$$
\mu\left(B_{i}\right)=B_{i}^{\prime} \text { for all } i, \quad \mu(\sigma)=\pi(\sigma), \quad \sigma \in K .
$$


Here the equality $f \circ \mu=f \circ \pi$ does not necessarily hold. So we need to modify $\mu$. By Lemma 11 we can modify it in a neighborhood of $\bigcup_{i} C_{i}$. To be precise, there exists a PL homeomorphism $\nu$ from a polyhedron neighborhood $V$ of $\bigcup_{i} C_{i}$ to a polyhedron neighborhood $V^{\prime}$ of $\bigcup_{i} C_{i}$ such that

$$
\begin{aligned}
& \nu=\mu \text { on } \bigcup_{i} C_{i}, \quad f \circ \nu=f \circ \pi \text { on } V, \\
& \nu\left(B_{i} \cap V\right)=B_{i}^{\prime} \cap V^{\prime} \quad \text { for all } i, \\
& \nu(\sigma \cap V)=\pi(\sigma) \cap V^{\prime}, \quad \sigma \in K .
\end{aligned}
$$

We want to extend $\nu$ to $\left|K^{k}\right| \cup V$ by induction on $k$. Hence it suffices to prove the following by the fact stated before the proof of the first half.

Let $Y$ be a cell, and let $f_{1}, f_{2}$ be PL functions on $Y$ such that

$$
0 \leq f_{1}, f_{2} \leq 3, \quad f_{1}^{-1}(0)=f_{2}^{-1}(0), \quad f_{1}^{-1}(3)=f_{2}^{-1}(3) .
$$

Set

$$
Y_{i}[a, b]=f_{i}^{-1}([a, b]) \quad \text { for } a<b \in \mathbf{R} \text { and } i=1,2 .
$$

Assume a PL homeomorphism

$$
\nu: \partial Y \cup Y_{1}[0,1] \cup Y_{1}[2,3] \rightarrow \partial Y \cup Y_{2}[0,1] \cup Y_{2}[2,3]
$$

such that

$$
f_{2} \circ \nu=f_{1} \quad \text { on } \partial Y \cup Y_{1}[0,1] \cup Y_{1}[2,3] .
$$

Assume also PL homeomorphisms

$$
\xi_{i}:(\text { a PL disk }) \times[1,2] \rightarrow Y_{i}[1,2], \quad i=1,2,
$$

such that $f_{i} \circ \xi_{i}(x, t)=t, i=1,2$. Then we can extend $\nu$ to a PL homeomorphism $\tau$ of $Y$ such that $f_{2} \circ \tau=f_{1}$.

Considering this statement on $Y_{1}[1,2]$ and $Y_{2}[1,2]$ only, moreover, we can reduce it to the following simpler one.

Let $Y_{1}, Y_{2}$ be $n$-simplexes. Let $\nu_{1}, \nu_{2}$ be a PL homeomorphism from $Y_{1}$ to $Y_{2}$ such that $\left.\nu_{1}\right|_{\partial Y_{1}}$ is PL isotopic to $\left.\nu_{2}\right|_{\partial Y_{1}}$. Then the isotopy can be extended to a PL isotopy from $\nu_{1}$ to $\nu_{2}$.

We prove easily this in the same way as the proof of the statement in the proof of Lemma 11. Thus we prove the latter half.

\section{Proofs OF THEOREMS}

Proof of Theorem 1. Existence. Assume $X$ to be noncompact. We can define by Lemma 4 subanalytic triangulations of $f$ on increasing compact subanalytic subsets of $X$. Combining these triangulations we want to define a subanalytic triangulation of $f$ on $X$. For that it suffices to prove the following.

Let $X_{1} \subset X_{2}$ be compact subanalytic subsets of $X$. Set

$$
Y=X_{1} \cap \overline{X_{2}-X_{1}} \text {. }
$$


Let $\left(K_{1}, \pi_{1}\right)$ be a subanalytic triangulation of $\left.f\right|_{X_{1}}$ such that $\pi_{1}^{-1}(Y)$ is the underlying polyhedron of a subcomplex $L$ of $K_{1}$. Then there exists a subanalytic triangulation $\left(K_{2}, \pi_{2}\right)$ of $\left.f\right|_{X_{2}}$ such that for every $\sigma \in K_{1}$ with $\sigma \cap Y=\phi$, $\pi_{2}^{-1} \circ \pi_{1}(\sigma)$ is a simplex in $K_{2}$ and $\left.\pi_{2}^{-1} \circ \pi_{1}\right|_{\sigma}$ is linear.

Proof of this statement. Assume $L$ to be full in $K_{1}$. (If $L$ is not so then replace $L$ by the biggest subcomplex of $K_{1}$ whose vertex set coincides with $L^{0}$.) Set

$$
N=N\left(L, K_{1}\right), \quad \partial N=\{\sigma \in N: \sigma \cap Y=\phi\} .
$$

By the first half of Lemma 4 there exists a subanalytic triangulation $\left(K_{2}^{\prime}, \pi_{2}^{\prime}\right)$ of $\left.f\right|_{\overline{X_{2}-X_{1}}}$ such that $\left\{\pi_{2}^{\prime}(\operatorname{Int} \sigma): \sigma \in K_{2}^{\prime}\right\}$ is compatible with $\left\{\pi_{1}(\sigma): \sigma \in L\right\}$. Let $L^{\prime}$ denote the subcomplex of $K_{2}^{\prime}$ with underlying polyhedron $=\pi_{2}^{\prime-1}(Y)$. We want to construct $K_{2}$ by pasting $K_{1}$ and $K_{2}^{\prime}$. For that we need to modify $\left.\pi_{2}^{-1} \circ \pi_{1}\right|_{Y}$ since it may be not PL.

Applying the latter half of Lemma 4 we obtain a PL homeomorphism $\nu$ from $|L|$ to $\left|L^{\prime}\right|$ such that

$$
\begin{aligned}
& f \circ \pi_{1}=f \circ \pi_{2}^{\prime} \circ \nu \text { on }|L|, \\
& \pi_{2}^{\prime-1} \circ \pi_{1}(\sigma)=\nu(\sigma) \text { for } \sigma \in L .
\end{aligned}
$$

Set

$$
\mu=\pi_{1}^{-1} \circ \pi_{2}^{\prime} \circ \nu
$$

Then $\mu$ is a subanalytic homeomorphism of $|L|$ such that

$$
\begin{aligned}
& f \circ \pi_{1} \circ \mu=f \circ \pi_{1} \text { on }|L|, \\
& \mu(\sigma)=\sigma \text { for } \sigma \in L .
\end{aligned}
$$

We will extend $\mu$ to $|N|$ holding these properties and the property

$$
\text { (4.|}|\partial N|) \quad \mu=\text { ident on }|\partial N| \text {. }
$$

Let $a_{1}, a_{2}, \ldots$ be the vertexes in $\partial N$, and let $N_{0}=L, N_{i}, i=1,2, \ldots$, be the biggest subcomplexes of $N$ such that $N_{i}^{0}=N_{i-1}^{0} \cup\left\{a_{i}\right\}$. We will construct extensions inductively on $\left|N_{i}\right|$. Assume a subanalytic extension homeomorphism $\mu_{i-1}$ of $\left|N_{i-1}\right|$ with $\left(2 .\left|N_{i-1}\right|\right),\left(3 . N_{i-1}\right)$ and (4.|$\left.\left|\partial N_{i-1}\right|\right)$, where $\partial N_{i-1}=\partial N \cap N_{i-1}$. Let $N_{i}^{\prime}$ be the subcomplex of $N_{i}$ generated by $\{\sigma \in$ $\left.N_{i}: \sigma \cap a_{i} \neq \phi\right\}$. Then we can assume $N_{i}=N_{i}^{\prime}$, i.e. $N_{i}$ is the cone with vertex $a_{i}$ and base $N_{i-1}$. Set

$$
\mu_{i}\left(t x+(1-t) a_{i}\right)=t \mu_{i-1}(x)+(1-t) a_{i}, \quad x \in\left|N_{i-1}\right|, 0 \leq t \leq 1 .
$$

Then $\mu_{i}$ is an extension of $\mu$ and a subanalytic homeomorphism of $\left|N_{i}\right|$ with $\left(2 .\left|N_{i}\right|\right),\left(3 . N_{i}\right)$ and $\left(4 .\left|\partial N_{i}\right|\right)$. Thus we obtain a subanalytic homeomorphism of $|N|$ with $(2 .|N|),(3 . N)$ and $(4 .|\partial N|)$. Let $\mu$ denote it also.

Replace $\pi_{1}$ by $\pi_{1}^{\prime}$ defined by

$$
\pi_{1}^{\prime}(x)= \begin{cases}\pi_{1}(x) & \text { for } x \in\left|K_{1}\right|-|N|, \\ \pi_{1} \circ \mu(x) & \text { for } x \in|N|\end{cases}
$$


Then $\pi_{2}^{\prime-1} \circ \pi_{1}^{\prime}$ is PL on $|L|$ by (1). Subdivide $L, L^{\prime}, K_{1}$ and $K_{2}^{\prime}$ so that $\pi_{2}^{\prime-1} \circ \pi_{1}^{\prime}$ is simplicial from $L$ to $L^{\prime}$ and $\left(K_{1}-N\right) \cup \partial N$ remains unsubdivided, and keep the same notations. Define $K_{2}$ as the quotient simplicial complex of the disjoint union of $K_{1}$ and $K_{2}^{\prime}$ by the equivalence relation of $\pi_{2}^{\prime-1} \circ \pi_{1}^{\prime}$, and define $\pi_{2}$ as $\pi_{1}^{\prime}$ on $\left|K_{1}\right|$ and as $\pi_{2}^{\prime}$ on $\left|K_{2}^{\prime}\right|$. Then $\left(K_{2}, \pi_{2}\right)$ satisfies the requirements in the above statement, which completes the proof of the existence.

Proof of uniqueness. Assume $X$ to be noncompact. For the same reason as the proof of the uniqueness in Lemma 4 we can assume that

$$
\left\{\pi^{\prime}\left(\text { Int } \sigma^{\prime}\right): \sigma^{\prime} \in K^{\prime}\right\}
$$

is compatible with $\{\pi(\sigma): \sigma \in K\}$. Let $K_{1} \subset K_{2} \subset \cdots, K_{1}^{\prime} \subset K_{2}^{\prime} \subset \cdots$ be sequences of finite full subcomplexes of $K, K^{\prime}$, respectively, such that

$$
\begin{array}{ll}
\bigcup_{i} K_{i}=K, \quad \bigcup_{i} K_{i}^{\prime}=K^{\prime}, \\
\pi^{\prime}\left(\left|K_{i}^{\prime}\right|\right)=\pi\left(\left|K_{i}\right|\right), & i=1,2, \ldots, \\
N\left(K_{i}, K\right) \subset K_{i+1}, & i=1,2, \ldots .
\end{array}
$$

Our construction of $\tau$ proceeds by stages on $\left|K_{i}\right|, i=1,2, \ldots$. For this we need only to prove the following.

Let $\tau_{2}$ be a PL homeomorphism from $\left|K_{2}\right|$ to $\left|K_{2}^{\prime}\right|$ such that

$$
\begin{aligned}
& f \circ \pi^{\prime} \circ \tau_{2}=f \circ \pi \quad \text { on }\left|K_{2}\right|, \\
& \tau_{2}(\sigma)=\pi^{\prime-1} \circ \pi(\sigma), \quad \sigma \in K_{2} .
\end{aligned}
$$

Then there exists a PL homeomorphism $\tau_{3}$ from $\left|K_{3}\right|$ to $\left|K_{3}^{\prime}\right|$ with $\left(1 .\left|K_{3}\right|\right)$, $\left(2 . K_{3}\right)$ and the property

$$
\tau_{3}=\tau_{2} \text { on }\left|K_{1}\right|
$$

We prove this in the same way as the proof of the existence. By Lemma 4 we have a PL homeomorphism $\nu$ from $\left|K_{3}\right|$ to $\left|K_{3}^{\prime}\right|$ with $\left(1 .\left|K_{3}\right|\right)$ and $\left(2 . K_{3}\right)$. We need to modify $\nu$ on $\left|K_{2}\right|$ so that (3) is satisfied. Set $\mu=\tau_{2}^{-1} \circ \nu$ on $\left|K_{2}\right|$. Then $\mu$ is a PL homeomorphism of $\left|K_{2}\right|$ such that

$$
f \circ \pi \circ \mu=f \circ \pi \quad \text { on }\left|K_{2}\right|, \quad \mu(\sigma)=\sigma, \quad \sigma \in K_{2} .
$$

The arguments in the proof of the existence imply a PL homeomorphism $\mu^{\prime}$ of $\left|K_{2}\right|$ such that

$$
\begin{aligned}
& f \circ \pi \circ \mu^{\prime}=f \circ \pi \text { on }\left|K_{2}\right|, \\
& \mu^{\prime}(\sigma)=\sigma, \quad \sigma \in K_{2}, \\
& \mu^{\prime}= \begin{cases}\text { ident } & \text { on }\left|K_{1}\right|, \\
\mu & \text { on } \overline{\left|K_{3}\right|-\left|K_{2}\right|} .\end{cases}
\end{aligned}
$$

Set

$$
\tau_{3}= \begin{cases}\tau_{2} \circ \mu^{\prime} & \text { on }\left|K_{2}\right| \\ \nu & \text { on }\left|K_{3}\right|-\left|K_{2}\right|\end{cases}
$$


Then $\tau_{3}$ satisfies the required properties. This proves Theorem 1 .

Remark 12. In Theorem 1 we can choose a subanalytic triangulation which is compatible with given subanalytic subsets of $X$ like Lemma 4. To be precise, let $\left\{Y_{i}\right\}$ be a locally finite family of subanalytic subsets of $X$. Then we can choose a subanalytic triangulation $(K, \pi)$ of $f$ so that $\{\pi(\operatorname{Int} \sigma): \sigma \in K\}$ is compatible with $\left\{Y_{i}\right\}$. The uniqueness holds true also in this case. Moreover we can require the condition that for every $\sigma$ in $K,\left.\pi\right|_{\operatorname{Int} \sigma}$ is of class analytic.

Proof. Only the last statement is not clear. We need to check the proofs of Lemma 8, the first half of Lemma 4 and of the existence of Theorem 1. Then the second proof is not sufficient for the present purpose. We modify the proof as follows.

Keep the same notations. We choose $\left(K_{1}, \pi_{1}\right)$ and $\left(K_{2}, \pi_{2}\right)$ (in the proof of Lemma 8) so that for every $\sigma$ in $K_{j}, j=1,2,\left.\pi_{j}\right|_{\text {Int } \sigma}$ is of class analytic. We wanted to paste $\left(K_{1}, \pi_{1}\right)$ and $\left(K_{2}, \pi_{2}\right)$. As an adhesive of this pasting we use a third subanalytic triangulation $\left(K_{3}, \pi_{3}\right)$ of $X[a]$ for $a=2.5$ such that $\left\{\pi_{3}(\operatorname{Int} \sigma): \sigma \in K_{3}\right\}$ is compatible with

$$
\bigcup_{j=1,2}\left\{\pi_{j}\left(\psi_{j}(\sigma \times a)\right): \sigma \in L_{j}\right\}
$$

and for every $\sigma \in K_{3},\left.\pi_{3}\right|_{\text {Int } \sigma}$ is of class analytic. Let $K_{1}^{\prime}, K_{2}^{\prime}$ be subdivisions of $\left.K_{1}\right|_{\pi_{1}^{-1}(X[0, a])},\left.K_{2}\right|_{\pi_{2}^{-1}(X[a, 5])}$, respectively, such that $\left.K_{1}^{\prime}\right|_{\pi_{1}^{-1}(X[a])},\left.K_{2}^{\prime}\right|_{\pi_{2}^{-1}(X[a])}$ are simplicially isomorphic to $L_{1} \times a, L_{2} \times a$ through $\psi_{1}, \psi_{2}$ respectively. Set

$$
\pi_{1}^{\prime}=\left.\pi_{1}\right|_{\left|K_{1}^{\prime}\right|}, \quad \pi_{2}^{\prime}=\left.\pi_{2}\right|_{\left|K_{2}^{\prime}\right|} .
$$

After modifying $\left(K_{1}^{\prime}, \pi_{1}^{\prime}\right)$ and $\left(K_{2}^{\prime}, \pi_{2}^{\prime}\right)$ in the same way as the proof of the existence of Theorem 1 so that

$$
\left.\pi_{3}^{-1} \circ \pi_{1}^{\prime}\right|_{\left.K_{1}^{\prime}\right|_{\pi_{1}^{\prime-1}(X|a|)}},\left.\quad \pi_{3}^{-1} \circ \pi_{2}^{\prime}\right|_{\left.K_{2}^{\prime}\right|_{\pi_{2}^{\prime-1}(X|a|)}}
$$

are simplicial to $K_{3}$, we paste $\left(K_{1}^{\prime}, \pi_{1}^{\prime}\right),\left(K_{3}, \pi_{3}\right)$ and $\left(K_{2}^{\prime}, \pi_{2}^{\prime}\right)$. Then the quotient simplicial complex is what we want.

Remark 13. Let $f$ be a subanalytic function on a polyhedron $X$. Then Theorem 1 and Lemma 10 imply a subanalytic homeomorphism $\pi$ of $X$ such that $f \circ \pi$ is PL. Moreover, if $f^{-1}(0)$ is a subpolyhedron of $X$ then we can choose $\pi$ to be the identity on $f^{-1}(0)$.

Proof of Corollary 2. By Remark 13 above we can assume that $f_{1}, f_{2}$ are PL functions on a polyhedron $X$. Then Corollary 2 follows from Lemma 11 .

Remark 14 (nonclosed case). Let $f$ be a subanalytic function on a subanalytic set $X$ in $\mathbf{R}^{n}$ (not necessarily closed in $\mathbf{R}^{n}$ ). Then there exist a simplicial complex $K$ and a subanalytic homeomorphism $\pi$ from a union of some open simplexes in $K$ to $X$ such that $f \circ \pi$ can be extended to $|K|$ and the restriction to every $\sigma \in K$ of the extension is linear. 
Proof. Let $Y$ denote the closure of the graph of $f$ in $\mathbf{R}^{n} \times \mathbf{R}$ and let $g$ denote the restriction to $Y$ of the projection $\mathbf{R}^{n} \times \mathbf{R} \rightarrow \mathbf{R}$. Then we can regard $X$ as a subanalytic subset of $Y$ and $f$ as the restriction to $X$ of $g$. Hence Remark 14 follows from Theorem 1 and Remark 12.

Remark 15 (semianalytic version). Let $f$ be a semianalytic function on a compact semianalytic set $X$. Then there exist a finite simplicial complex $K$ and a semianalytic homeomorphism $\pi$ from $|K|$ to a neighborhood of $f^{-1}(0)$ in $X$ such that for every $\sigma \in K,\left.f \circ \pi\right|_{\sigma}$ is linear.

Indeed the semianalytic case of Lemma 8 is sufficient for this, and the proof of Lemma 8 proceeds also in this case. We need only note the following and Lemma 16. Let $V$ be a semianalytic bounded set in $\mathbf{R}^{n} \times \mathbf{R}$, and let $p$ be the projection $\mathbf{R}^{n} \times \mathbf{R} \rightarrow \mathbf{R}^{n}$. Assume that $V$ is described by a finite number of analytic functions $f_{i}(x, t)$ (i.e. $\left.V=U \cap\left(\left\{f_{i}=0\right\} \cap\left\{f_{i^{\prime}}>0\right\} \cap\left\{f_{i^{\prime \prime}}<0\right\}\right)\right)$ which are polynomial in $t$. Then $p(V)$ is semianalytic (e.g. [5]).

It seems very possible to extend the above result to the same form as Theorem 1. However I do not know its proof.

Lemma 16. Let $A \subset \mathbf{R}^{n} \times \mathbf{R}$ be a bounded semianalytic set. Set

$$
\begin{aligned}
& B=\left\{\lambda \in S^{n-1}: A\right. \text { is locally described by a finite number of } \\
& \text { analytic functions } f_{i}(y, z, t),(y, z, t) \in \mathbf{R}^{n-1} \times \mathbf{R} \times \mathbf{R} \\
&=\mathbf{R}^{n} \times \mathbf{R}, \text { which are polynomial in } z \text {, where the coordinate } \\
&\left.\quad \text { system of } \mathbf{R}^{n} \text { is linearly changed so that } \mathbf{R} \lambda \text { is the } z \text {-axis }\right\}, \\
& C=S^{n-1}-B .
\end{aligned}
$$

Then $C$ is contained in a subanalytic set $C^{\prime}$ of dimension $<n-1$.

Proof. Consider the problem around 0 in $\mathbf{R}^{n} \times \mathbf{R}$, and let $A$ be described by a finite number of nonzero analytic functions $f_{i}(x, t)$. We can assume that every $f_{i}$ vanishes at 0 and is irreducible as an alytic function germ at 0 . Denote by $A_{i}$ for each $i$ the zero set of $f_{i}$, and define a set $A_{i 0}$ in $\mathbf{R}^{n}$ by $A_{i 0} \times 0=A_{i} \cap \mathbf{R}^{n} \times 0$. If $A_{i} \supset\{t=0\}$ then $f_{i}$ is the multiple of $t$ and a function nonvanishing at 0 , hence we can disregard $f_{i}$. Therefore we can assume that all $A_{i 0}$ are of dimension $<n$. For a point $\lambda$ of $S^{n-1}$ nonsingular for $A_{i 0}$ at $0 \in \mathbf{R}^{n}$, change linearly the coordinate system of $\mathbf{R}^{n}$ so that $\mathbf{R} \lambda$ is the $n$th axis. Then by Weierstrass preparation theorem we can replace $f_{i}$ by an analytic function $g(y, z, t),(y, z, t) \in \mathbf{R}^{n-1} \times \mathbf{R} \times \mathbf{R}=\mathbf{R}^{n} \times \mathbf{R}$, which is polynomial in $z$. Consequently

$$
C \subset \bigcup_{i}\left\{\lambda \in S^{n-1}: \text { singular for } A_{i 0} \text { at } 0\right\} \text {. }
$$

Hence Lemma 16 follows from Lemma 6.

Proof of Theorem 3. If $Y$ is compact we prove this theorem in the same way as Lemma 4. So consider the noncompact case. By the stereographic projection 
$\mathbf{R}^{n} \rightarrow S^{n}$ we can assume that $Y$ is contained in $S^{n} \subset \mathbf{R}^{n+1}$. Then the closure of the graph of $f$ in $\mathbf{R}^{n+1} \times \mathbf{R}$ is compact. Hence Theorem 3 follows in the same way as Remark 14.

Problem 17. Let $\varphi$ be a holomorphic function on a complex manifold $M$. Do there exist a simplicial complex $K$ and a real semianalytic homeomorphism $\pi$ from $|K|$ to $M$ such that for every $\sigma \in K,\left.\varphi \circ \pi\right|_{\sigma}$ is linear? How can we extend Theorem 1 to the subanalytic map case?

\section{REFERENCES}

1. B. Giesecke, Simpliziable Zerlegung abzählbarer analytische Räume, Math. Z. 83 (1964), 177213.

2. R. M. Hardt, Triangulation of subanalytic sets and proper light subanalytic maps, Invent. Math. 38 (1977), 207-217.

3. H. Hironaka, Subanalytic sets, Number Theory, Algebraic Geometry and Commutative Algebra, in honour of Y. Akizuki, Kinokuniya, Tokyo, 1973, pp. 453-493.

4. __ Triangulation of algebraic sets, Proc. Sympos. Pure Math., vol. 29, Amer. Math. Soc., Providence, R. I., 1975, pp. 165-185.

5. S. Lojasiewicz, Triangulation of semi-analytic sets, Ann. Scuola Norm. Sup. Pisa 18 (1964), 449-474.

6. J. W. Milnor, Two complexes which are homeomorphic but combinatorially distinct, Ann. of Math. 74 (1961), 575-590.

7. J. R. Munkres, Elementary differential topology, Ann. of Math. Studies, no. 54, Princeton Univ. Press, 1963.

8. C. P. Rourke and B. J. Sanderson, Introduction to piecewise-linear topology, Ergeb. Math., Bd. 69, Springer, 1976.

9. M. Shiota, Piecewise linearization of real analytic functions, Publ. RIMS Kyoto Univ. 20 (1984), 727-792.

10. M. Shiota-M. Yokoi, Triangulations of subanalytic sets and locally subanalytic manifolds, Trans. Amer. Math. Soc. 286 (1984), 727-750.

11. B. Teissier, Sur la triangulation des morphismes sous-analytic, preprint.

Department of Mathematics, College of General Education, Nagoya University, NAGOYA, JAPAN 